\title{
THE D-MODULE STRUCTURE OF F-SPLIT RINGS
}

\author{
KAREN E. SMITH
}

The purpose of this note is to point out an interesting connection between the structure of a commutative, Noetherian ring of prime characteristic as a (left) module over its ring of differential operators and various well studied properties such as F-purity, F-regularity, and strong F-regularity. Theorem 2.2 establishes the first connections between the D-module structure of rings of characteristic $p$ and the theory of tight closure introduced by Hochster and Huneke [HH1]. This theorem can also be viewed as a partial answer to a characteristic $p$ version of the question raised by Levasseur and Stafford [LS, 0.13.3]: "When is $R$ a simple $D(R)$ module?" For $F$-split rings $R$ of low dimension, the answer is that $R$ is simple as a $D(R)$ module if and only if all ideals of $R$ are tightly closed. In the last section, connections with pseudorational local rings are discussed, and some of the difficulty in passing between the characteristic zero and characteristic $p$ cases is indicated.

Throughout this note, $R$ will denote a reduced, commutative, Noetherian ring of prime characteristic $p>0$. The $\operatorname{ring} R$ is assumed to be a finitely generated algebra (or equivalently, module) over its subring $R^{p}$ of $p^{t h}$ powers. This mild assumption implies that $R$ is excellent $[\mathrm{K}]$, and is satisfied, for instance, whenever $R$ is finitely generated over a perfect field or is a complete local ring with a perfect residue field. The symbol $I$ denotes an arbitrary ideal of $R$, and for any such $I$, the symbol $I^{[q]}$ denotes the ideal of $R$ generated by the $q^{\text {th }}$ powers of the elements (equivalently, the generators) of $I$, where $q=p^{e}$ is an integer power of $p$.

\section{Tight closure and test ideals}

Test ideals are central to the study of tight closure. A variant notion of the idea of a test ideal for $R$ defined by Hochster and Huneke in [HH1] will be introduced. First we summarize some of the terminology associated with the study of tight closure.

Received May 31, 1995.

The author is partially supported by the NSF. 
Let $I$ be any ideal of $R$. The tight closure $I^{*}$ of $I$ is the ideal of $R$ consisting of all those elements $z \in R$ for which there exists some $c \in R$ not in any minimal prime of $R$ such that

$$
c z^{q} \in I^{[q]} \text { for all } q=p^{e}>>0 .
$$

An ideal is said to be tightly closed if $I^{*}=I$. Rings in which all ideals are tightly closed are of particular interest. Such rings are called (weakly) Fregular. All weakly F-regular rings are Cohen-Macaulay and normal [HH1] and even have rational singularities [S1]. The use of the adjective "weakly" is supposed to indicate that it is not known whether a localization of a weakly F-regular ring is weakly F-regular. The unqualified term F-regular is reserved for those weakly F-regular rings all of whose localizations are also weakly F-regular.

The ring $R$ is strongly $F$-regular if, for all $c$ not in any minimal prime of $R$, there exists some $q$ such that the $R$-linear map $R \longrightarrow R^{1 / q}$ sending 1 to $c^{1 / q}$ splits as a map of $R$ modules. The property of strong F-regularity is preserved under localization; it is easy to see that regular rings are strongly F-regular and that strongly F-regular rings are F-regular [HH2]. Strong and weak F-regularity may turn out to be equivalent; this is known to be the case for Gorenstein rings [HH2] and rings of dimension less than or equal to three [Wil].

Two properties closely related to F-regularity which are known to behave well under localization are $F$-splitting and $F$-rationality. The ring $R$ is said to be $F$-split if the map $R^{p} \hookrightarrow R$ splits as a map of $R^{p}$ modules. (When $R$ is a finitely generated $R^{p}$ module, as it always is in this paper, F-split is equivalent to $F$-pure which means that the map $R^{p} \hookrightarrow R$ remains injective upon tensoring with any $R^{p}$ module.) Note that F-splitting is equivalent to the condition expressed above for strong F-regularity where $c$ is taken to be the identity element of the ring. In particular, $R \hookrightarrow R^{1 / q}$ splits for some $q=p^{e}$ if and only if it splits for all $q=p^{e}$. The ring $R$ is said to be $F$-rational if every ideal generated by parameters is tightly closed; a set of $k$ elements of $R$ is called a set of parameters if it generates an ideal of height $k$. F-rational rings, like F-regular rings, are Cohen-Macaulay and normal [HH2], and have rational singularities [S1]. If $R$ is Gorenstein, then F-rationality, weak F-regularity, and strong F-regularity are all equivalent [HH2]. There are F-rational rings that are not F-regular, however [Wat].

An important feature of the definition of tight closure is that the element $c$ in (1.1) may depend on both $I$ and $z$, but not on $q$. Of course, the dependence of $c$ on $z$ is rather superficial, since there are always elements $c$ for which (1.1) holds for all $z$ in $I^{*}$. The dependence on $I$ is more 
fundamental. For a fixed ideal $I$ of $R$, the set of elements $c \in R$ such that (1.1) holds for each $z \in I^{*}$ forms an ideal of $R$. We call this the test ideal for $I$, and denote it by $\tau(I)$. More precisely, we define the test ideal for $I$ as

$$
\tau(I)=\bigcap_{q>>0} I^{[q]}:_{R}\left(I^{*}\right)^{[q]}
$$

where the intersection over $q>>0$ is interpreted as follows. For any collection of ideals $\left\{J_{q}\right\}_{q \in \Lambda}$, indexed by an increasing sequence of integers $\Lambda$, the collection of ideals $\left\{\cap_{t \geq q} J_{t}\right\}_{q \in \Lambda}$ is also indexed by $\Lambda$ and forms an increasing chain of ideals of $R$; since $R$ is Noetherian, this chain stabilizes and this stable ideal is denoted by $\bigcap_{q>>0} J_{q}$. An element $c$ in $\tau(I)$ but not in any minimal prime of $R$ will be called a test element for $I$.

For any collection of ideals $\mathcal{I}$ in a ring $R$, we can define the test ideal of this collection of ideals to be the ideal of all elements which can be used in "tight closure tests" for all ideals in the collection $\mathcal{I}$; that is $\tau(\mathcal{I})=$ $\bigcap_{I \in \mathcal{I}} \tau(I)$. It is not at all clear that we should expect this intersection to be non-trivial for arbitrary collections of ideals in $R$. A result of Hochster and Huneke that lies at the heart of the usefulness of tight closure is the remarkable fact that indeed there exist elements which are test elements for every ideal of $R$. In particular, any element which is in the defining ideal of the singular locus for Spec $R$ has a power with this property [HH2]. The test ideal for all ideals of $R$ will be denoted by $\tau$.

The reader is cautioned that the use of the words "test ideal" and "test element" in this paper differs slightly from that in [HH1] in that here we are concerned only with what happens for $q$ large. For an analysis of the test ideal for the collection $\mathcal{I}$ of ideals generated by a system of parameters, see [S2]. We will show here that every test ideal (for an ideal or a collection of ideals) is a submodule of $R$ regarded as a left module over its ring of differential operators.

\section{Differential operators and tight closure}

Let $A$ be an arbitrary commutative ring and $R$ be any commutative $A$ algebra. The ring of $A$ linear differential operators of $R$ is a certain (non-commutative) subring of $\operatorname{End}_{A}(R)$ defined inductively as follows: the zero $^{\text {th }}$ order differential operators are simply the $R$-linear endomorphisms of $R$, which we identify with $R$. An operator $\theta \in \operatorname{End}_{A}(R)$ is a differential operator of order $\leq k \in \mathbb{N}$ if, for all $r \in R=\operatorname{End}_{R}(R)$, the commutator $[\theta, r]=\theta \circ r-r \circ \theta$ is a differential operator of order $\leq k-1$. By definition, $R$ is naturally a left $D_{A}(R)$ module. We say that $R$ is $D$-simple if it is simple when regarded as a left $D(R)$ module, that is, when the only ideals 
of $R$ stable under the action of all differential operators are the zero and the unit ideal. For basic facts on differential operators, the reader is referred to [EGA, IV, 16].

In our set up, $R$ is a reduced, commutative, Noetherian ring with the property that $R$ is finitely generated over its subring of $p^{t h}$ powers. We take $A=\mathbb{Z}$ and drop the subscript $A$ from the notation. With these assumptions, it is not hard to show ([Yek]) that

$$
D(R)=\bigcup_{e \in \mathbb{N}} \operatorname{End}_{R^{p^{e}}}(R) .
$$

We can now prove the results connecting tight closure with differential operators.

\section{Theorem 2.2.}

(1) Every test ideal of $R$ is a $D(R)$ submodule of $R$.

(2) If $R$ is simple as a $D(R)$ module, then for each ideal $I$ of $R$, there exists $q=p^{e}$, such that $I^{*}=I R^{1 / q} \cap R$.

(3) If $R$ is simple as a $D(R)$ module and if $U \subset R$ is any multiplicative system, then $\left(I U^{-1} R\right)^{*}=I^{*}\left(U^{-1} R\right)$. That is, tight closure behaves well under localization in $D$-simple rings.

(4) $R$ is strongly F-regular if and only if $R$ is F-split and is a finite product of D-simple rings.

Proof.

(1) It is clear that an arbitrary intersection of $D(R)$ submodules of $R$ is a $D(R)$ submodule of $R$, so it suffices to show that $\tau(I)$ is a $D(R)$ submodule of $R$, where $I$ is a fixed arbitrary ideal of $R$. Fix a set of generators $x_{1}, x_{2}, \ldots, x_{d}$ for $I$. Let $c$ be any element of $\tau(I)$. We need to show that for any $\theta \in D(R)$, the image of $c$ under $\theta$ is also in $\tau(I)$. We may assume that $\theta$ is $R^{q_{0}}$ linear, and hence $R^{q}$ linear for all $q>q_{0}$. Because $c \in \tau(I)$, we know that for each $z \in I^{*}$ and each $q>>0$, there exist elements $a_{1 q}, a_{2 q}, \ldots, a_{d q}$ of $R$ such that

$$
c z^{q}=a_{1 q} x_{1}^{q}+a_{2 q} x_{2}^{q}+\cdots+a_{d q} x_{d}^{q} .
$$

Thus, provided that $q \geq q_{0}$, we see that

$$
\theta(c) z^{q}=\theta\left(a_{1 q}\right) x_{1}^{q}+\theta\left(a_{2 q}\right) x_{2}^{q}+\cdots+\theta\left(a_{d q}\right) x_{d}^{q} .
$$


It follows that for all $\theta \in D(R)$ and all $c \in \tau(I), \theta(c)$ is in $\tau(I)$, whence $\tau(I)$ is a $D(R)$ submodule of $R$.

(2) Suppose that $R$ is D-simple. Consider the test ideal $\tau(I)$ for any fixed arbitrary $I \subset R$. By (1), $\tau(I)$ is a $D(R)$ submodule of $R$; it must therefore be either the zero ideal or the unit ideal of $R$. However, no test ideal $\tau(I)$ can ever be the zero ideal. Indeed, if $1 \notin \tau(I)$, then then there is some $z \in I^{*}-I$ and there is some $c$ not in any minimal prime such that $c z^{q} \in I^{[q]}$ for all $q>>0$. So $c \in \tau(I)$ and thus $\tau(I)$ is nonzero.

It follows that $\tau(I)$ must be the unit ideal. In particular, $1 \in$ $I^{[q]}: I^{*[q]}$, whence $I^{*[q]} \subset I^{[q]}$ for all $q>>0$. Taking $q^{\text {th }}$ roots, we see $I^{*} \subset I R^{1 / q} \cap R$. Since the reverse inclusion is obvious, we conclude that $I^{*}=I R^{1 / q} \cap R$.

(3) The inclusion $I^{*} U^{-1} R \subset\left(I U^{-1} R\right)^{*}$ is clear in general. Let $R^{\infty}=$ $\bigcup_{q=p^{e}} R^{1 / q}$ be the ring of all $q^{\text {th }}$ roots of $R$. The preceding paragraph shows that $I^{*}=I R^{\infty} \cap R$ for all ideals $I$ of $R$. For any multiplicative system $U \subset R$, note that $U^{-1}\left(R^{\infty}\right)=\left(U^{-1} R\right)^{\infty}$. It easily follows that $\left(I U^{-1} R\right)^{*}=I\left(U^{-1} R\right)^{\infty} \cap U^{-1} R=I U^{-1} R^{\infty} \cap U^{-1} R=$ $U^{-1}\left(I R^{\infty} \cap R\right)=I^{*}\left(U^{-1} R\right)$. This completes the proof that tight closure behaves well under localization in D-simple rings.

(4) Because $R$ is strongly F-regular if and only if it is a finite product of strongly F-regular domains [HH2], the statement in both directions reduces to the statement that a domain is strongly F-regular if and only if it is F-split and D-simple.

Assume that $R$ is an F-split D-simple domain. From (2), it follows immediately that $\mathrm{D}$-simple, $\mathrm{F}$-split rings are $\mathrm{F}$-regular, since D-simple implies that $I^{*}=I R^{1 / q} \cap R$ and F-split implies that $I R^{1 / q} \cap R=I$ for any ideal in $R$. However, unless weak and strong F-regularity turn out to be equivalent in general, the assertion in (3) claims a stronger conclusion.

To see that $R$ is in fact strongly F-regular, let $c$ be any nonzero element of $R$. We claim that there exists $q$ such that the $R$-linear map $R \longrightarrow R^{1 / q}$ defined by $1 \mapsto c^{1 / q}$ splits. Because $R$ is a simple $D(R)$ module, the $D$ submodule generated by $c$ must be all of $R$. In particular, there exists some $\theta \in D(R)$ such that $\theta(c)=1$. Suppose that $\theta$ is $R^{q}$ linear for some $q=p^{e}$. Because $R$ is Fsplit, the map $R^{q} \hookrightarrow R$ splits as a map of $R^{q}$ modules for all $q$. Let $\pi \in \operatorname{Hom}_{R^{q}}\left(R, R^{q}\right)$ denote such a splitting and consider the map $\psi=\pi \circ \theta \in \operatorname{Hom}_{R^{q}}\left(R, R^{q}\right)$. We see that $\psi(c)=1$ and that $\psi$ is isomorphic to the $R$-linear map $\psi^{1 / q}: R^{1 / q} \longrightarrow R$ sending $r^{1 / q} \mapsto\{\psi(r)\}^{1 / q}$. Note that $\psi^{1 / q}$ sends $c^{1 / q} \in R^{1 / q}$ to $1 \in R$. 
This is the desired splitting of the map $R \longrightarrow R^{1 / q}$ showing that $R$ is strongly F-regular.

To prove the converse, suppose that $R$ is a strongly F-regular domain. Taking $c=1$ in the definition of strong F-regularity, we see that $R$ is obviously $\mathrm{F}$-split.

To see that a strongly F-regular domain $R$ is a simple $D$ module, it suffices to show that each nonzero element of $R$ generates all of $R$ under the action of $D(R)$. Let $c$ be any nonzero element of $R$. We know that there exists $q$ such that the $R$ linear map $R \longrightarrow R^{1 / q}$ sending 1 to $c^{1 / q}$ splits. Let $\theta \in \operatorname{Hom}_{R}\left(R^{1 / q}, R\right)$ be any such splitting. The map $\theta$ gives rise to a differential operator $\theta^{q} \in \operatorname{End}_{R^{q}}(R)$ defined as follows: $\theta^{q}$ sends an element $r \in R$ to $\left(\theta\left(r^{1 / q}\right)\right)^{q} \in R^{q} \subset R$. We see that this differential operator takes $c$ to 1 , whence the $D(R)$ module generated by $c$ is all of $R$. This shows that every strongly F-regular domain is D-simple. This concludes the proof.

A remark is in order regarding (2) above. The question of whether tight closure is well behaved under localization has been a persistent open problem. Although (2) solves this problem for a special class of rings, in fact the class of D-simple rings is poorly understood. All the examples of Dsimple rings known to the author are either already F-regular or otherwise known to have trivial tight closure properties. In fact, I do not know any examples of a normal D-simple ring which is not F-regular.

2.3. Corollary. Let $R$ be an F-split domain. Assume that the dimension of $R$ is no greater than three or that $R$ is Gorenstein. The ring $R$ is simple as a module over its ring of differential operators if and only if all ideals of $R$ are tightly closed.

Proof. Strong F-regularity is equivalent to the property that all ideals are tightly closed for rings of dimension three or less [Wil] and for Gorenstein rings [HH2]. Thus the corollary is immediate from $(2.2,(4))$.

\section{Rational singularities}

Theorem 2.2 implies that F-split, D-simple rings are pseudorational (i.e., have rational singularities, suitably interpreted in prime characteristic, see $[\mathrm{LT}])$. This is a consequence of the fact that F-rational rings are pseudorational [S1].

It is possible that for F-split, Gorenstein rings having rational singularities is equivalent to D-simplicity. Such an equivalence would follow from an affirmative answer to the question: "Does a pseudorational local ring 
have some ideal generated by a system of parameters tightly closed?" It is worth noting that this question makes sense also in characteristic zero and may well be true in characteristic zero even if it fails in general in characteristic $p>0$. The question of the equivalence of F-rationality and pseudorationality has been raised throughout the tight closure literature (see, for example, [HH2], [FW], or [S1]). It is known for graded complete intersection rings with an isolated singularity (at least when the characteristic $p$ is large relative to the degrees of the defining equations) $[\mathrm{FW}]$ and for graded rings of dimension two $[\mathrm{F}]$. This dimension two case can also be accomplished using differential operators [S3].

It can not be the case that D-simplicity is equivalent to pseudorationality for general non-Gorenstein rings, however. Watanabe gave an example of a two dimensional F-split rational singularity which has a non-tightly closed ideal [Wat]. Theorem 2.2 implies that this ring can not be D-simple.

There is a D-module analog of Boutôt's theorem for rational singularities.

Proposition 3.1. Let $S$ and $T$ be arbitrary commutative A algebras, where $A$ is a commutative ring. Suppose that $S \hookrightarrow T$ and that this inclusion splits as a map of $S$ modules. If $T$ is a simple $D_{A}(T)$ module, then $S$ is a simple $D_{A}(S)$ module.

Proof. Let $c \in S$ be any nonzero element. We need to find an element $\theta \in$ $D_{A}(S)$ such that $\theta(c)=1$. Because $T$ is D-simple, there exists $\psi \in D_{A}(T)$ such that, considering $c$ as an element of $T, \psi(c)=1$. Let $i$ denote the inclusion $S \hookrightarrow T$ and let $\pi$ be any $S$ module splitting of $i$. Consider the $A$ linear endomorphism of $S, \theta=\pi \circ \psi \circ i$. It is clear that $\theta(c)=1$, so we need only check that $\theta \in D(S)$.

We proceed by induction on the order of $\psi$. If $\psi$ is of order zero, then $\psi$ is multiplication by some element $t \in T$. In this case, $\theta$ is easily seen to be multiplication by the element $\pi(t) \in S$, whence $\theta$ is an order zero differential operator on $S$. Now suppose that for all differential operators on $T$ of order strictly less than $k$, the corresponding element in $\operatorname{End}_{A}(S)$ is a differential operator on $S$ of the same order or less. Assume $\psi \in D(T)$ has order $k$. For all $s \in S$, note that

$$
\begin{aligned}
{[\theta, s] } & =[\pi \circ \psi \circ i, s] \\
& =\pi \circ \psi \circ i \circ s-s \circ \pi \circ \psi \circ i \\
& =\pi \circ \psi \circ s \circ i-\pi \circ s \circ \psi \circ i \\
& =\pi \circ(\psi \circ s-s \circ \psi) \circ i \\
& =\pi \circ([\psi, s]) \circ i .
\end{aligned}
$$


Because $[\psi, s]$ is a differential operator on $T$ of order less than $k$, the inductive assumption guarantees that $[\theta, s]$ is a differential operator of order less than $k$ on $S$. This says that $\theta$ is a differential operator on $S$ of order less than or equal to $k$, and the proof is complete.

The issue of a possible relationship between rings of differential operators and rational singularities has been raised before. Levasseur and Stafford pointed out a possible connection between rational singularities of an algebraic variety over a field of characteristic zero with the property that the ring of differential operators on it is a simple ring [LS]. The condition that $D(R)$ is a simple ring is a strictly stronger condition than the condition that $R$ is a simple $D(R)$ module, as pointed out in [LS]. However, it is not clear how much stronger this condition is; rings of differential operators are difficult to compute and the only $\mathrm{D}$-simple rings $R$ known to this author for which $D(R)$ is not simple are non-normal. Likewise, it is possible that the F-split condition may be replaced by something weaker in Theorem 2.2(4).

Theorem 2.2 sheds some light on characteristic $p$ versions of some questions asked by Levasseur and Stafford. For example, Question 0.13.1 of [LS] asks what additional hypotheses are needed to ensure that the ring of differential operators on a pseudorational local ring has nice properties. The results of this paper indicate that (in characteristic $p$ ), an F-rational, Gorenstein local ring is at least simple as a D-module. Indeed, if the conjecture that F-rationality and pseudorationality are equivalent turns out to be true, then (at least in characteristic $p$ ) rational singularities will be equivalent to the presence of both D-simplicity and F-purity for Gorenstein rings. For non-Gorenstein rings, the condition that $R$ is D-simple and F-split is strictly stronger than the condition that $R$ has rational singularities, in the same way that the condition that all ideals are tightly closed is stronger than the condition that the subclass of parameter ideals is tightly closed. It seems likely that Gorensteinness will play a key role in answering the characteristic zero question of Levasseur and Stafford.

Although the characteristic $p$ results may shed some light on what we may expect to find in characteristic zero, the issue of drawing precise conclusions about finitely generated algebras over a field of characteristic zero by reduction to characteristic $p$ seems to be a tricky business. In general, there are "more" differential operators in characteristic p. We briefly sketch below a situation where this is the case.

Let

$$
\begin{aligned}
R & =\frac{\mathbb{C}[X, Y, Z]}{\left(X^{3}+Y^{3}+Z^{3}\right)}, \\
R_{\mathbb{Z}} & =\frac{\mathbb{Z}[X, Y, Z]}{\left(X^{3}+Y^{3}+Z^{3}\right)} .
\end{aligned}
$$


It is not hard to check that the natural map

$$
D_{\mathbb{Z}}\left(R_{\mathbb{Z}}\right) \otimes_{\mathbb{Z}} \mathbb{C} \longrightarrow D_{\mathbb{C}}(R)
$$

is an isomorphism. However, when $p$ is congruent to 1 modulo 3 , the map

$$
D_{\mathbb{Z}}\left(R_{\mathbb{Z}}\right) \otimes_{\mathbb{Z}} \mathbb{Z} / p \mathbb{Z} \longrightarrow D_{\mathbb{Z} / p \mathbb{Z}}\left(R_{\mathbb{Z}} \otimes_{\mathbb{Z}} \mathbb{Z} / p \mathbb{Z}\right)
$$

is never an isomorphism, even on an open set of Spec $\mathbb{Z}$. (With some work, one can see that, after localizing at a single element of $\mathbb{Z}$, this map is injective for all $p$; this aspect of the reduction to characteristic $p$ program does hold in general.)

The reason that the above map fails to be surjective is that $\bar{R}=R_{\mathbb{Z}} \otimes$ $\mathbb{Z} / p \mathbb{Z}$ is F-split when $p$ is congruent to 1 modulo 3. (see Example 7.16 in [HH4], or note that the splitting is equivalent to an injective Frobenius action on $H_{(x, y, z)}^{2}(R)$, which is in turn equivalent to the requirement that the corresponding elliptic curve is not supersingular and the claim follows from exercise IV, 4.14 in [Ha]). There therefore exists an $\bar{R}^{p}$ linear map splitting the inclusion $\bar{R}^{p} \hookrightarrow \bar{R}$. Such a splitting, $\theta$, can be interpreted as a differential operator on $\bar{R}$, since it lies in $\operatorname{Hom}_{\bar{R}^{p}}\left(\bar{R}, \bar{R}^{p}\right) \subset \operatorname{Hom}_{\bar{R}^{p}}(\bar{R}, \bar{R}) \subset$ $D(\bar{R})$. It is not hard to check that there must be such a splitting $\theta$ which preserves the degrees of $\bar{R}$, that is, we can find an element of the $\mathbb{Z}$ graded ring $D(\bar{R})$ which has degree zero and sends $\bar{R}$ to $\bar{R}^{p}$. If $(*)$ were to be an isomorphism, $\theta$ would lift to an operator in $D_{\mathbb{Z}}\left(R_{\mathbb{Z}}\right)$, also of degree zero, say $\theta^{\prime}$.

The operators in $D_{\mathbb{C}}\left(R_{\mathbb{C}}\right)$ of degree zero are completely described in [BGG]; they are generated (as a $\mathbb{C}$ algebra) by the Euler operator $X \frac{\partial}{\partial X}+$ $Y \frac{\partial}{\partial Y}+Z \frac{\partial}{\partial Z}$. In particular, each degree zero operator will send a homogeneous element $f$ to $f$ multiplied by some polynomial function in the degree of $f$. Thus $\theta^{\prime}$ can be described by a particular polynomial $P$ which takes integer values on the integers: $\theta^{\prime}$ sends each homogeneous element $f$ to $P(\operatorname{deg}(f)) f$. Because $\theta^{\prime}$ reduces "mod $p$ " to the F-splitting $\theta$, we see that $P\left(\operatorname{deg}\left(f^{p}\right)\right)=1 \bmod p$. But then for any element $g$ of degree a multiple of $p$ which is not a $p^{t h}$ power, we also have that $\theta(g)=\{P(\operatorname{deg}(g)) \bmod p\} g=$ $g$, contrary to the fact that the image of $\theta$ is $R^{p}$.

As the example above shows, "reduction to characteristic $p$ " for rings of differential operators is likely to be difficult in general. It is worth pointing out that it does work for some rings, for example, polynomial rings.

\section{References}

[BGG] Bernstein, Gelfand, and Gelfand, Differential operators on the cubic cone, Russian Mathematical Surveys 27 (1972), 169-174. 
[F] R. Fedder, A Frobenius characterization of rational singularity in two dimensional graded rings, Trans. Amer. Math. Soc. 340 (1993), 655-668.

[FW] R. Fedder and K. Watanabe, A characterization of F-Regularity in terms of FPurity, MSRI Publications No. 15 (1989), Springer-Verlag, New York, 227-245.

[EGA] A. Grothendieck and J. Dieudonné, Elements of Algebraic Geometry, IV, Publ. Math, IHES 32 (1967).

[Ha] R. Hartshorne, Algebraic Geometry, vol. 52, Springer-Verlag, Graduate Texts in Mathematics, New York, 1987.

[HH1] M. Hochster and C. Huneke, Tight closure, invariant theory, and the BriançonSkoda theorem, Jour. Amer. Math. Soc. 3 (1990), 31-116.

[HH2] , F-regularity, test elements and smooth base change, Trans. Amer. Math. Soc. 346 (1994), 1-62.

[HH3] _ Tight closure and strong F-regularity, Mémoires de la Société Mathématique de France 38 (1989), 119-133.

[HH4] , Tight closure of parameter ideals and splitting in module finite extensions, Jour. of Algebraic Geometry 3 (1994), 599-670.

[K] E. Kunz, On Noetherian rings of characteristic p, Amer. J. Math. 91 (1976), 999-1031.

[LS] T. Levasseur and T. Stafford, Rings of differential operators on classical rings of invariants, Memoirs of the AMS 412 (1989).

[LT] J. Lipman and B. Tessier, Pseudorational local rings and a theorem of BriançonSkoda on the integral closures of ideals, Mich. Math. J. 28 (1981), 97-116.

[S1] K. E. Smith, F-rational rings have rational singularities, preprint.

[S2] - Test ideals in local rings, Trans. Amer. Math. Soc. (to appear).

[S3] - Tight closure in graded rings, preprint.

[Wat] K. I. Watanabe, F-regular and F-pure normal graded rings, J. Pure Appl. Algebra 71 (1991), 341-350.

[Wil] L. Williams, Uniform stability of kernels of Frobenius, Jour. of Algebra 172 (1995), 721-743.

[Yek] A. Yekutieli, An explicit construction of the Grothendieck residue complex, Asterique 208 (1992).

Department of M athematics, M it , Cambridge M A 02139

E-mail address: kesmith@math.mit.edu 